\title{
Dissociated effects of botulinum toxin chemodenervation on ocular deviation and saccade dynamics in chronic lateral rectus palsy
}

\author{
J F Acheson, C R Bentley, J Shallo-Hoffmann, M A Gresty
}

\begin{abstract}
Aim-Changes in saccade velocityl amplitude characteristics (main sequence) and attenuation of distance esotropia in response to botulinum toxin (BTX-A) chemodenervation of the antagonist medial rectus were studied in a group of nine patients with chronic lateral rectus palsy.

Methods-Serial measurements of ocular deviation and infrared oculograms of saccadic eye movements to targets at $5^{\circ}-20^{\circ}$ of lateral gaze were made before injection and at $2,4,8,16$, and 20 weeks after injection.

Results-At 2 weeks after injection, the ocular deviation changed by a mean of 34.5 prism dioptres and the $5^{\circ}$ and $10^{\circ}$ adduction saccades were significantly slowed $(p<0.02$ Wilcoxon signed rank test). By the second examination, however, the adducting saccade peak velocity had returned to normal while the mean ocular deviation remained significantly changed $(p=0.01$ Wilcoxon matched pairs). By 20 weeks the mean ocular deviation was not significantly different from that before injection $(p=0.14$ matched pairs).

Conclusions-The ocular realignment caused by BTX-A may persist after saccadic function has been restored. This may be because toxin may have a more profound and long lasting effect on the orbital singly innervated fibres which are active tonically at rest to hold gaze whereas there is relative sparing of the additional motor units recruited during fast eye movements.

(Br F Ophthalmol 1998;82:67-71)
\end{abstract}

Since the introduction of botulinum toxin into the management of strabismus by Scott, ${ }^{1}$ a number of investigators have reported the results of treatment in patients with sixth nerve (abducens) palsy..$^{2-5}$ Treatment of acute patients is probably unnecessary as many recover spontaneously and intervention may confuse neurological assessment in a potentially progressive clinical problem. ${ }^{6}$ In those patients who do not recover spontaneously after 4-6 months, however, botulinum toxin chemodenervation has been shown to be of value in the assessment and rehabilitation of binocular function. ${ }^{2}$ The denervation of the antagonist medial rectus may lead to a persistent, or more usually, a transient improvement in the angle of distance esotropia. Among those patients in whom the effect is transient, the clinician can assess residual lateral rectus function and so choose between horizontal rectus recess/resect procedures and vertical rectus transposition. For those patients in whom there is a persistent ocular realignment, extraocular muscle surgery may be avoided. ${ }^{7}$

It remains unclear whether a persistent effect from botulinum chemodenervation is dose dependent and by what mechanism a permanent ocular realignment is achieved when (and if) it occurs. Four patients with partial lateral rectus paresis treated by Elston and $\mathrm{Lee}^{8}$ enjoyed a persistent restoration of fusion, high grade stereopsis, and an expanded field of binocular single vision long after muscle reinnervation would be expected to have taken place. That the original lateral rectus weakness had failed to recover is documented by persistent abduction deficit in three out of four patients. Repka et al ${ }^{5}$ found that $32 \%$ of a group of 22 patients had restoration of binocular single vision in the primary position. Possible mechanisms for this effect include permanent structural changes in the denervated muscle, ${ }^{9}{ }^{10}$ a central effect within the brainstem, ${ }^{11}$ release or prevention of contracture development, ${ }^{12}$ and altered sensory homeostasis.

The purpose of this study was to evaluate the effects on ocular motility of a single dose challenge of botulinum toxin on ocular deviation. Tropic changes were studied as the main determinant of symptomaticity. In addition, since saccadic eye movements recruit an additional population of orbital muscle fibres from those involved in position holding, saccade dynamics were assessed to gain insight into the differential response of orbital muscle fibre groups to toxic denervation.

\section{Methods}

SUBJECTS

Four male and five female patients (see Table 1), age range 23-82 years (mean 54.6 years) with chronic ( $>6$ months) abducens nerve palsy were recruited from the neuroophthalmology clinic at the National Hospital and the motility clinic at the Western Eye Hospital, London. All patients had agreed to a trial botulinum toxin chemodenervation to assess residual lateral rectus function and consented to the additional observations made in this study according to the guidelines of the local ethics committee. Before inclusion patients had ophthalmological and neurological assessments.
Accepted for publication 13 August 1997 


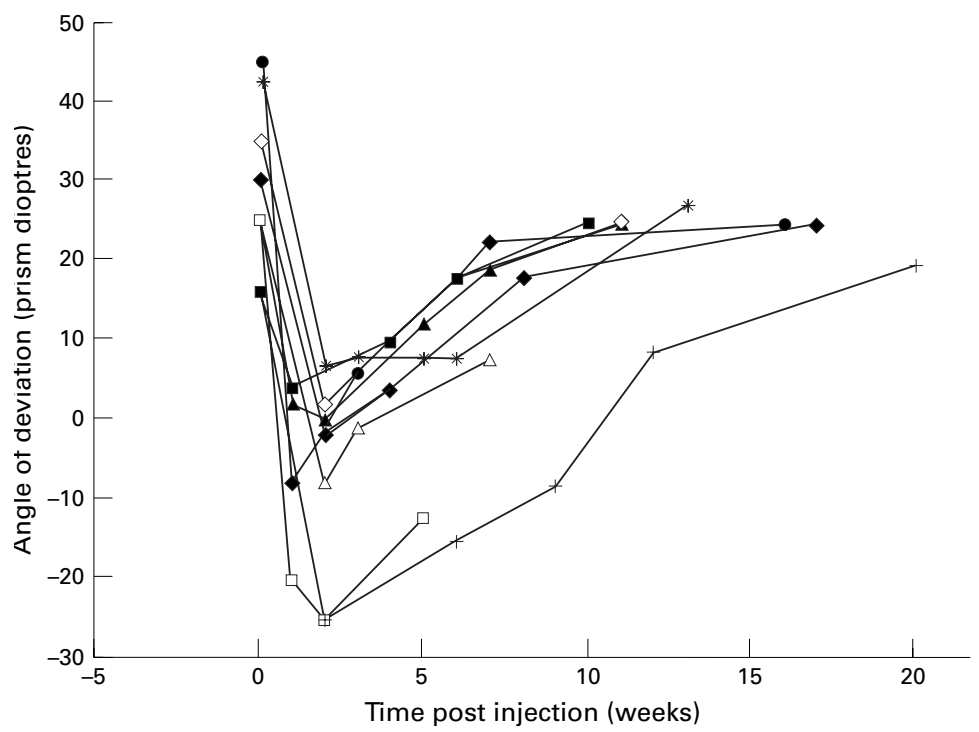

Figure 1 Changes over time in the angle of deviation for each subject following injection of botulinum toxin measured in prism dioptres.

Table 1 Normal group

\begin{tabular}{lll}
\hline Amplitude (degrees) & Adduction peak velocity & Abduction peak velocity \\
\hline 5 & $218.7(46.6)$ & $222.8(19.1)$ \\
10 & $314.4(55.6)$ & $314.9(37.6)$ \\
15 & $370.7(69.7)$ & $374.4(74.6)$ \\
20 & $390.6(67.1)$ & $395.8(58.8)$ \\
\hline
\end{tabular}

Mean peak velocities measured in degrees/second for adducting and abducting saccades in a group of normal individuals (SD).

Eye movement recordings were also taken from a control group of nine healthy subjectsfour male, five female, age range 23-71 years (mean 47.4 years).

PROTOCOL

Orthoptic assessment

Pretreatment ocular deviation was measured using cover test, prism cover test and duction ranges were plotted on a Hess chart from the Lees screen.

Oculography

All patients had eye movement recordings made before and after toxin for both abduction and adduction in both affected and normal eyes. Eye movement recordings were made using an infrared limbal reflection system (DC bandwidth $500 \mathrm{~Hz}$, Skalar Instruments). The patient was seated with a head brace and chin rest $100 \mathrm{~cm}$ from a centrally viewed red-light emitting diode target. Patients were required to make a minimum of 10 saccades to command between 5 and 20 degrees in 5 degree increments, under monocular viewing conditions for each direction of eye movement. Cali- bration of each eye was performed under monocular conditions. In the case of the paretic eye, calibration saccades into the field of paresis were performed only as far as accurate recordings were possible.

\section{Treatment}

Each patient received 3.75 units of botulinum toxin A (Dysport) diluted in $0.15 \mathrm{ml}$ of normal saline and injected into the ipsilateral medial rectus muscle under topical anaesthesia (amethocaine drops $1 \%$ ) using a 27 gauge unipolar hollow needle (Allergan) under electromyographic guidance.

\section{Timing of assessments}

Subjects were studied with both orthoptic measures and eye movement recordings before injection and at 1 week after injection and if no adduction deficit could be demonstrated a further injection was performed (only required in one subject). Further assessments were then made at 1 or 2 weekly intervals over a mean follow up of 4 months.

\section{SACCADE ANALYSIS}

Eye movements were analysed off line. In this study only saccades away from the primary position (that is, centrifugal saccades) were included, in order to study active muscle function, rather than passive elastic restoring forces. Amplitudes were plotted against peak velocities for both abduction and adduction for all analysable saccades within the study period of 1 minute for each amplitude. Main sequences were generated using computerised non-linear regression analysis ${ }^{13}$ fitting the exponential function:

$$
\mathrm{PV}=\mathrm{V}_{\max }\left(1-\mathrm{e}^{-\mathrm{A} / \mathrm{C}}\right)
$$

(where PV = peak velocity, $\mathrm{V}_{\max }=$ asymptotic velocity, $\mathrm{A}=$ amplitude, and $\mathrm{C}=$ constant).

\section{STATISTICAL ANALYSIS}

For statistical analysis the movements were divided into amplitude bins ${ }^{14}$ of $5^{\circ}, 10^{\circ}, 15^{\circ}$, and $20^{\circ}$ to reduce the variability introduced by individual fluctuations in saccade amplitude. Normal subjects were compared initially with the patient groups (Wilcoxon-Mann-Whitney for independent samples). Similarly pre- and postinjection data were compared (Wilcoxon signed ranks test for related or matched samples).

\section{Results}

ORTHOPTIC MEASURES

Changes in the angle of deviation following toxin and recovery are summarised in Figure 1. The mean deviation at 6 metres fixation before

Table 2 Adduction peak velocities pretoxin and at first post-toxin visit

\begin{tabular}{llllll}
\hline & \multicolumn{2}{l}{ Affected eye } & & \multicolumn{2}{c}{ Unaffected eye } \\
\cline { 2 - 3 } \cline { 5 - 6 } Amplitude (degrees) & Pretoxin & Post-toxin & & Pretoxin & Post-toxin \\
\hline 5 & $218.7(30.5)$ & $162.7(74)$ & & $223(51)$ & $231(39)$ \\
10 & $333.4(52)$ & $250.3(101)$ & & $304.8(43)$ & $327.5(76)$ \\
15 & $369.3(72)$ & $295(128.4)$ & & $347.6(66.7)$ & $355(65)$ \\
20 & $433(98)$ & $425 / 346^{\star}$ & & $410.5(85)$ & $385.6(89)$ \\
\hline
\end{tabular}

Mean peak velocities in degrees/second for adducting saccades pre and post-toxin.

${ }^{\star}$ Only two subjects were able to achieve 20 degrees of adduction post-toxin. 
Table 3 Abduction peak velocities pretoxin and at first post-toxin visit

\begin{tabular}{llllll}
\hline & \multicolumn{2}{l}{ Affected eye } & & \multicolumn{2}{l}{ Unaffected eye } \\
\cline { 2 - 3 } \cline { 5 - 6 } Amplitude (degrees) & Pretoxin & Post-toxin & & Pretoxin & Post-toxin \\
\hline 5 & $223(46)$ & $215.8(63)$ & & $223.7(51)$ & $256.5(29)$ \\
10 & $308.7(40)$ & $280.7(142)$ & & $318.7(47)$ & $342.3(118)$ \\
15 & $355.7(52)$ & $328.7(146.8)$ & & $362(58)$ & $398.7(102)$ \\
20 & $296 / 235$ & $348 / 456^{\star}$ & & $465.8(122)$ & $410.6(110)$ \\
\hline
\end{tabular}

Mean peak velocities in degrees/second for abducting saccades pre and post-toxin.

${ }^{\star}$ Only two subjects were able to achieve 20 degrees of abduction post-toxin.

injection was 29 prism dioptres (PD). Immediately following injection (1-2 weeks) the squint improved to a mean deviation of -5.5 (SD 10.9) PD; a mean change of 34.5 PD, significant at $\mathrm{p}<0.05$ (Wilcoxon matched pairs). By 20 weeks the mean deviation for the group as whole had returned to $24 \mathrm{PD}$ (SD 4.3) of esotropia. This was not significantly different for the group as a whole ( $p=0.14 \mathrm{Wil}-$ coxon match pairs) from the pretoxin deviation. Six patients returned to within 10 PD of the original deviation after 4 months. Three patients had a persistent reduction in deviation at 4 months.

\section{SACCADES}

Peak saccade velocities for abduction and adduction are shown for normal subjects in Table 1. Saccade velocities for patients' affected and good eyes pre and post-toxin are shown in Tables 2 and 3 respectively. The following results highlight the major relevant findings.

Lateral rectus function (abduction)

As a group, the peak velocities of abduction in patients' squinting eyes were significantly slower for $15^{\circ}$ and $20^{\circ}$ saccades than normal $\mathrm{p}<0.05$ (Wilcoxon signed ranks) and had not changed significantly at the first assessment after injection (that is, 1-2 weeks).

\section{Medial rectus function (adduction)}

Before injection the peak velocities of adducting saccades were normal in all subjects. When assessed at the first visit following toxin chemo-



Figure 2 The course of recovery of 5 degree saccade dynamics following botulinum toxin injection. Saccade peak velocity is measure in degrees/second, deviation is measured in prism dioptres. denervation of the medial rectus seven subjects had impaired medial rectus function. One subject failed to achieve even $5^{\circ}$ saccades and two could not make $10^{\circ}$ adductions.

Where comparisons were possible, peak velocities were found to be significantly slowed for the eight subjects who could make $5^{\circ}$ saccades and the seven subjects who could make $10^{\circ}$ saccades $\mathrm{p}<0.02$ (Wilcoxon signed ranks). Of the five subjects that could attain $15^{\circ}$ adducting saccades three had normal velocity for $5^{\circ}$ and $10^{\circ}$ saccades with slowing at $15^{\circ}$ (as estimated from the SD of normal range) and, surprisingly, the other two had slow small amplitude saccades but normal velocity $15^{\circ}$ saccades. Saccades were of normal velocity at all amplitudes in the only two subjects who could make $20^{\circ}$ saccades.

At the second post-toxin assessment adducting saccades were of normal peak velocity. The course of recovery of saccade dynamics following toxin is shown in Figure 2.

Saccades of the unaffected eye

The main sequences for both abducting and adducting saccades in the patients' unaffected eyes assessed before treatment and on the first visit post-toxin were normal.

\section{Discussion}

The effect of medial rectus chemodenervation was an immediate reduction in distance esotropia, which was maximal at the first visit (1-2 weeks) the mean change was 34.5 PD. This compares well with the literature. Fitzsimons et $\mathrm{al}^{3}$ found an average change of $28 \mathrm{PD}$ in a group of unilateral sixth nerve patients using a high dose regimen of $0.1 \mathrm{ml}\left(6.25 \times 10^{-5}\right.$ $\mu \mathrm{g}$ Dysport or $3.12 \times 10^{-4}$ Allergan). Repka et $a l^{5}$ reported a mean change of $16 \mathrm{PD}$ after a single injection (18 PD whole group success and failures, multiple injections) but overall averaged 1.7 injections per patient (of between 2.5 and 7.5 units). Elston $\mathrm{et} a l,{ }^{15}$ in a large group of strabismic adults, with a range of diagnoses, found a mean of $34 \mathrm{PD}$ change. Others have reported varying levels of response. ${ }^{58}$

The peak velocity for abduction in the affected eye before injection was low in comparison with controls and with abduction velocities of the unaffected eye. This was to be expected. Metz et al ${ }^{16}$ observed this qualitatively in 12 patients, commenting that eye movement recordings would be required to detect abnormality in cases of mild paresis where the velocity reduction is relatively small: Zee and $\mathrm{Yee}^{17}$ subsequently confirmed this was the case. 
Our subjects had long standing paresis, of a minimum duration of 6 months (mean 30 months) who might be expected to have developed tonic medial rectus overaction referred to as medial rectus contracture. ${ }^{11}$ One might expect medial rectus contracture to slow abduction saccades and possibly affect the dynamics of adduction. Of note, in our study abducting peak velocities did not change following botulinum toxin and, pretoxin, the velocity of adducting saccades in the affected eyes were normal. These findings raise questions about whether "contracture" opposes and slows movements of the antagonist muscle and, if so, under what circumstances. For example, it could be that the effects of a contracture of the medial rectus become apparent in more extreme positions of gaze than can be tested with infrared oculography. ${ }^{5}$

The marked reduction in adducting velocities apparent at the first post injection visit was not maintained on subsequent follow up. In contrast, orthoptic measures at the second visit were still significantly different $p=0.01$ (Wilcoxon matched pairs). There is thus a dissociation between the two variables of ocular motility which point to a difference in the mechanisms underlying the changes in saccade peak velocity and the changes in deviation. This effect has been suggested by earlier investigators: Magoon and Scott ${ }^{18}$ noted in 1982 that after botulinum chemodenervation a paralysed muscle with only $10-20 \%$ preinjection force may still generate near normal saccade velocities and ocular rotations.

Possible explanations for the dissociation include saccadic adaptation, differences in the effects of botulinum toxin on extraocular muscle fibre subpopulations, and sarcomere augmentation:

(a) Saccadic adaptation-an increase in the magnitude of the centrally generated saccadic pulse to the denervated adductor could possibly restore normal adduction. This pulse would have to be uniocular since the other eye did not increase its corresponding velocity but we are unaware of any supporting physiological evidence for this possibility. If the increase in pulse were bilateral the corresponding abductor should show a large pulse step mismatch which was not the case.

(b) Dissociated effects of botulinum toxin on extraocular muscle subpopulations-The current view of the functional organisation of extraocular muscle fibres is that all participate in saccades but that the orbital singly innervated fibres are implicated in gaze holding and are the first to be recruited with slow phase movements around the primary position. ${ }^{19} 20$ Although all fibres may be initially affected, botulinum toxin was shown to have the longest lasting (permanent) effect on the orbital singly innervated fibres in a monkey model studied by Spencer and $\mathrm{McNeer} .{ }^{9}$ Therefore, we may expect that the vergence tone of the injected adductor can be permanently weakened so that improvement in ocular alignment is maintained. In contrast, because the saccades recruit all other fibre groups which are not so affected by toxin, their velocity characteristics would only suffer a tran- sient impairment of any significance with only minimal chronic slowing due to loss of the orbital singly innervated fibres.

(c) A further possible explanation comes from interesting experimental animal data showing that sarcomeres can be added to the stretched medial rectus muscle when the globe is sutured into an exotropic position. ${ }^{10}$ The lengthened medial rectus would be able to generate normal saccades with a new ocular alignment as a result of this adaptation. However, it remains to be shown that this pathological response to forcible muscle stretching is also a consequence of botulinum injection and how it might relate to acquired strabismus.

There was considerable intersubject variability in the response of the injected medial rectus when assessed on the first postoperative occasion: this probably reflects differential effects of toxin on different fibre populations in the extraocular muscle. For the two patients who had normal velocities at all saccade amplitudes tested, we assumed that population of motor units specifically recruited for saccades had been little affected by toxin, although it had had a measurable effect on their deviation. In the case of the patients who could attain only $15^{\circ}$ but managed normal velocities at this amplitude, smaller saccades being slower, we assume that the additional muscle units recruited for this size of saccade were unaffected by toxin and generated the main driving force for the eye movement so that slowing was minimal.

\section{Conclusions}

We have shown in a prospective study on the effect of botulinum toxin chemodenervation of the medial rectus that saccade velocities are slowed in the immediate postinjection phase in the "on" direction for the injected muscle and return to preinjection values in 2-4 weeks, unlike ocular deviation which is chronically attenuated. Saccade dynamics and orthoptic variables involve different oculomotor functions which may depend on the specific sensitivities of the various types of orbital muscle fibres to toxin and their differential recovery from denervation.

1 Scott AB. Botulinum toxin injection as an alternative to strabismus surgery. Ophthalmology 1980;87:1044-9.

2 Scott AB, Kraft P. Botulinum toxin injection in the management of lateral rectus paresis. Ophthalmology 1985; 92:676-83.

3 Fitzsimons R, Lee JP, Elston J. Treatment of sixth nerve palsy in adults with combined botulinum toxin chemodenervation and surgery. Ophthalmology 1988;95:1535-42.

4 Biglan AW, Burnstine RA, Rogers GL, Saunders RA. Management of strabismus with botulinum A toxin. Ophthalmology 1989;96:935-43.

5 Repka MX, Lam GC, Morrison NA. The efficacy of botulinum neurotoxin A for the treatment of complete and partially recovered chronic sixth nerve palsy. F Pediatr Ophthalmol Strabismus 1994;31:79-83.

6 Lee J, Harris S, Cohen J, et al. Results of a prospective randomised trail of botulinum toxin therapy in acute unilateral sixth nerve palsy. $f$ Pediatr Ophthalmol Strabismus 1994;31:283-6.

7 Riordan-Eva P, Lee JP. Management of VIth nerve palsyavoiding unnecessary surgery. Eye 1992;6:386-90.

8 Elston JS, Lee JP. Paralytic strabismus: the role of botulinum toxin. Br F Ophthalmol 1985;69:891-6.

9 Spencer RE, McNeer KW. Botulinum toxin paralysis of adult monkey extraocular muscle. Structural alterations in orbital singly innervated muscle fibers. Arch Ophthalmol 1987;105:1703-11.

10 Scott AB. Change of eye muscle sarcomeres according to eye position. F Pediatr Ophthalmol Strabismus 1994;31:85-8. 
11 Wiegand $\mathrm{H}$, Wellhoner HH. The action of botulinum A neurotoxin on the inhibition by by antidromic stimulation of the lumbar monosynaptic reflex. Naunyn Schmiedebergs Arch Pharmacol 1977;298:235-8.

12 Kommerell G, Olivier D. Kontrakturen nach Augenmuskelparesen Albrecht Von Graefes Arch Klin Exp Ophthalmol 1971;183:169-78.

13 Baloh RW, Sills AW, Kumley WE, Honrubia V. Quantitative measurement of saccadic amplitude, duration and velocity. Neurology 1975;25:1065-70.

14 Barton JJS, Sharpe JA. Saccadic jitter is a quantitative ocular sign in myasthenia gravis. Invest Ophthalmol Vis $\mathrm{Sci}$ 1995;36:1566-72.

15 Elston JS, Lee JP, Powell CM, Hogg C, Clark P. Treatment of strabismus in adults with botulinum toxin A. $\mathrm{Br} \mathcal{F} \mathrm{Oph}$ - thalmol 1985;69:718-24.

16 Metz HS, Scott AB, O'Meara D, Stewart HL. Ocular saccades in lateral rectus palsy. Arch Ophthalmol 1970;84: 453-60.

17 Zee DS, Yee RD. Abnormal saccades in paralytic strabismus. Am f Ophthalmol 1977;83:112-4.

18 Magoon EH, Scott AB. Eye muscle paralysis: relationship of muscle force to ocular rotation and saccadic velocity. Invest Ophthalmol Vis Sci 1982;22:s266.

19 Robinson DA. The functional behaviour of the peripheral ocular motor apparatus: a review. In: Kommerell G, ed. Disorders of ocular motilty. Munich: Bergman, 1978:43-61.

20 Scott AB, Collins CC. Division of labour in human extraocular muscle. Arch Ophthalmol 1973;90:319-22. 